\title{
Replication study for the association of
}

\section{rs391300 in SRR and rs17584499 in PTPRD with susceptibility to type 2 diabetes in a Japanese population}

\author{
Minako Imamura ${ }^{1 *}$, Minoru Iwata ${ }^{2,3}$, Hiroshi Maegawa ${ }^{4}$, Hirotaka Watada ${ }^{5,6}$, Hiroshi Hirose ${ }^{7}$, Yasushi Tanaka ${ }^{8}$, \\ Kazuyuki Tobe ${ }^{2}$, Kohei Kaku ${ }^{9}$, Atsunori Kashiwagi ${ }^{4}$, Takashi Kadowaki ${ }^{10}$, Ryuzo Kawamori ${ }^{6}$, Shiro Maeda ${ }^{1}$
}

\begin{abstract}
Aims/Introduction: Genetic risk variants for type 2 diabetes; rs391300-G in SRR and rs17584499-T in PTPRD, have been identified by a genome-wide association study using Han Chinese individuals living in Taiwan. In an attempt to know the effects of these two variants in conferring susceptibility to type 2 diabetes in the Japanese, we carried out a replication study for the association of the two single nucleotide polymorphisms (SNPs) with type 2 diabetes in a Japanese population.

Materials and Methods: We genotyped 11,530 Japanese individuals (8,552 type 2 diabetes patients and 2,978 controls) for rs391300 and rs17584499, and analyzed the association of these two SNPs with type 2 diabetes by logistic regression analysis.

Results: Neither of the variants was associated with susceptibility to type 2 diabetes in the Japanese population (rs391300-G: odds ratio $[\mathrm{OR}]=0.97 ; 95 \%$ confidence interval $[\mathrm{Cl}]$ 0.91-1.04; $P=0.44$; rs17584499-T: $\mathrm{OR}=1.04 ; 95 \% \mathrm{Cl} 0.96-1.14 ; P=0.34)$. Adjustment or stratified analysis for age, sex and body mass index (BMI) did not affect the association of these variants with the disease. We did not observe a significant association of the SNPs with any metabolic traits, BMl, fasting plasma glucose, homeostasis model assessment of $\beta$-cell function (HOMA- $\beta$ ) and HOMA of insulin resistance (HOMA-IR) $(P>0.05)$.
\end{abstract}

Conclusions: Neither rs391300 nor rs17584499 had a significant effect on conferring susceptibility to type 2 diabetes in the Japanese population. (J Diabetes Invest doi: 10.1111/jdi.12017, 2013)

\section{KEY WORDS: Genetic association studies, Japanese, Type 2 diabetes mellitus}

\section{INTRODUCTION}

A genome-wide association study (GWAS) is a powerful method to detect genetic variations that predispose to a disease $^{1,2}$. The first round of GWAS for type 2 diabetes was reported by several European and American groups in $2007^{3}$, and approximately 60 type 2 diabetes susceptibility loci have been identified so far. The majority of them have been initially detected in populations of European descent, and several loci including TCF7L2, CDKAL1, HHEX, SLC30A8, KCNJ11,

\footnotetext{
Laboratory for Endocrinology and Metabolism, RIKEN Center for Genomic Medicine, Yokohama, ${ }^{2}$ First Department of Internal Medicine, ${ }^{3}$ Community Medical Support Unit, Faculty of Medicine, University of Toyama, Toyama, ${ }^{4}$ Department of Medicine, Shiga University of Medical Science, Otsu, Shiga, ${ }^{5}$ Department of Medicine, Metabolism and Endocrinology, School of Medicine, ${ }^{6}$ Sportology Center, Graduate School of Medicine, Juntendo University, ${ }^{7}$ Health Center, Keio University School of Medicine, ${ }^{10}$ Department of Diabetes and Metabolic Diseases, Graduate School of Medicine, The University of Tokyo, Tokyo, ${ }^{8}$ Division of Metabolism and Endocrinology, Department of Internal Medicine, St. Marianna University School of Medicine, Kawasaki, Kanagawa, and ${ }^{9}$ Division of Diabetes, Endocrinology and Metabolism, Department of Internal Medicine, Kawasaki Medical School, Kurashiki, Okayama, Japan

*Corresponding author. Minako Imamura Tel.: +81-45-503-9595 Fax: +81-45-503-9567 E-mail address: mimamura@src.riken.jp

Received 23 April 2012; revised 26 July 2012; accepted 7 September 2012
}

$C D K N 2 A / B, I G F 2 B P 2, G C K R$ and IRS1 have been confirmed as type 2 diabetes risk loci also in Japanese populations ${ }^{4-11}$. Four loci were identified by Japanese GWAS, and subsequent replication studies showed that three loci, KCNQ1 $1^{4,5}, C 2 C D 4 A$ $C 2 C D 4 B^{6}$ and $A N K 1^{12}$, were common susceptibility loci for type 2 diabetes across East Asian and European populations, whereas the association of UBE2E2 might be specific to East Asian populations ${ }^{6}$; suggesting the existence of genetic heterogeneity among different ethnicities.

In 2010, four additional genetic risk variants for type 2 diabetes were identified by two independent Han Chinese GWAS. Two variants, rs10906115 in CDC123/CAMK1D and rs1359790 near SPRY2, were discovered by Shu et al. ${ }^{13}$, and Tsai et al. identified another two, namely rs391300 in SRR and rs17584499 in PTPRD ${ }^{14}$. The association of rs10906115 and rs1359790 with type 2 diabetes was replicated in a Japanese population ${ }^{15}$. Meanwhile, the association of rs391300 or rs17584499 with type 2 diabetes in Japanese populations has not yet been clarified.

In the present study, we aimed to evaluate the contribution of these two variants, rs391300 in SRR and rs17584499 in PTPRD, to conferring susceptibility to type 2 diabetes in the Japanese. 


\section{MATERIALS AND METHODS}

\section{Participants, DNA preparation and genotyping}

DNA samples were prepared from peripheral blood of Japanese patients with type 2 diabetes $(n=8,552)$ who were enrolled in BioBank Japan or recruited from the outpatient clinics of the Shiga University of Medical Science, Kawasaki Medical School, St. Marianna University, University of Toyama and Juntendo University. We also examined 2,978 individuals (controls) who were enrolled from an annual health check carried out at Keio University or St. Marianna University, from the outpatient clinics of Toyama University or from the Japanese general population registered in the Japanese single nucleotide polymorphism (SNP) database. Control participants with glycated hemoglobin $\left(\mathrm{HbA}_{1 c} ;\right.$ National Glycohemoglobin Standardization Program [NGSP]) $\geq 6.4 \%^{16}$, fasting plasma glucose $\geq 7 \mathrm{mmol} / \mathrm{L}$ or selfreported diabetes were excluded from the present study.

Diabetes was diagnosed according to the criteria established by the World Health Organization ${ }^{17}$. Type 2 diabetes is clinically defined as a disease with gradual onset in adults. Individuals who tested positive for anti-glutamic acid decarboxylase antibodies and those diagnosed as having mitochondrial disease (mitochondrial myopathy, encephalopathy, lactic acidosis and stroke-like episodes [MELAS]) or maturity onset diabetes of the young were not included in the present study. Obesity was defined as body mass index (BMI) $\geq 25$, according to the criteria for obesity disease in Japan ${ }^{18}$.

Written informed consent was obtained from all the participants, and anonymity of the participants was preserved during the study. DNA was extracted using the standard phenol-chloroform procedure. The study protocol conformed to the provisions of the Declaration of Helsinki, and was approved by the ethics committee of the RIKEN Yokohama Institute and of each of the participating institutes. As shown in Table 1, type 2

Table 1 | Clinical characteristics of the participants

\begin{tabular}{lll}
\hline & Type 2 diabetes & Control \\
\hline Sample size & 8,552 & 2,978 \\
Male/female & $5,240 / 3,312$ & $1,656 / 1,322$ \\
Age (years) & $63.6 \pm 11.1$ & $50.5 \pm 16.2$ \\
Duration (years) & $11.8 \pm 9.2$ & - \\
Age at diagnosis (years) & $52.2 \pm 12.3$ & - \\
Systolic BP (mmHg) & $134 \pm 17$ & $128 \pm 18$ \\
Diastolic BP (mmHg) & $76 \pm 12$ & $78 \pm 12$ \\
HbA 1 (NGSP; \%) & $7.9 \pm 2.3$ & $5.3 \pm 0.4$ \\
FPG (mg/dL) & $152.0 \pm 54.7$ & $95.2 \pm 10.9$ \\
TC (mg/dL) & $199.4 \pm 35.3$ & $205.5 \pm 33.2$ \\
TG (mg/dL) & $127.0 \pm 73.6$ & $105.6 \pm 75.3$ \\
HDL-C (mg/dL) & $55.7 \pm 17.0$ & $63.2 \pm 16.0$ \\
BMl (kg/m $\left.{ }^{2}\right)$ & $24.2 \pm 4.0$ & $22.8 \pm 3.2$ \\
\hline
\end{tabular}

Data are means \pm standard deviation. BMl, blody mass index; BP, blood pressure; FPG, fasting plasma glucose; $\mathrm{HbA}_{1 c}$, glycated hemoglobin; HDL-C, high-density lipoprotein cholesterol; NGSP, National Glycohemoglobin Standardization Program; TC, total cholesterol; TG, triglyceride. diabetes cases had a higher BMI than controls $(24.2 \pm 4.0$ vs $22.8 \pm 3.2)$, and the cases were older than the controls $(63.6 \pm 11.1$ vs $50.5 \pm 16.2)$. These values were adjusted during subsequent analysis, if necessary. Genotyping of each SNP was carried out by a multiplex-polymerase chain reaction (PCR) invader assay as described previously ${ }^{19}$. The DNA sequence for oligo primers for PCR, and invader probes and signal probes are described in Table S1. The accuracy of the genotyping was confirmed by success rates of the assays (rs391300 98.7\%, rs17584499 98.2\%) and the concordance rates (100\%) in 101 $(0.88 \%$ of sample size in the present study) randomly selected duplicated samples.

The association data around the rs391300 locus and rs17584499 locus were obtained from our previously reported GWAS data ${ }^{12}$ for 4,470 type 2 diabetes cases (3,000 overlapped with the present study) and 3,071 independent controls. Only data with high imputation quality, proper info $>0.8$ and minor allele frequency $(\mathrm{MAF})>0.01$ were included in this analysis.

\section{Statistical analysis}

We carried out Hardy-Weinberg equilibrium (HWE) tests according to the method described by Nielsen et al. ${ }^{20}$ To test the association of each SNP with disease susceptibility, the differences between the case and control groups in terms of the distribution of genotypes, scored using an additive model (0, 1 and 2 for homozygous for non-effect allele, heterozygous and homozygous for effect allele, respectively), dominant model (0 for homozygous for non-effect allele, and 1 for heterozygous and homozygous effect allele) and recessive model ( 0 for homozygous for non-effect and heterozygous allele, and 1 for homozygous effect allele) were analyzed using logistic regression analysis with or without adjusting for age, sex and log-transformed BMI.

Quantitative trait analyses for BMI, fasting plasma glucose (FPG; 1,332 controls), homeostasis model assessment of $\beta$-cell function (HOMA- $\beta$; 900 controls) and HOMA of insulin resistance (HOMA-IR; 900 controls) were carried out by multiple linear regression analysis with or without adjusting for age, sex and log-transformed BMI. As the present Japanese samples have skewed distribution for values of BMI, FPG, HOMA-IR and HOMA- $\beta$, we carried out the analyses for the quantitative traits using log-transformed-BMI, -FPG, -HOMA-IR and HOMA- $\beta$. These analyses were carried out using JMP9 software (SAS Inc. Japan, Tokyo). Power calculation was carried out with a CaTS power calculator (CaTS: http://www.sph. umich.edu/csg/abecasis/CaTS/). Combined meta-analysis was carried out by the DerSimonian-Laird random effects model after evaluating homogeneity of effect size across studies by Cochran's Q statistics.

\section{RESULTS}

The genotype distribution of rs391300 and rs17584499 in control individuals did not deviate from HWE (Table 2). Neither rs391300 nor rs17584499 was significantly associated with 
Table 2 | Associations of rs391300 and rs17584499 with yype 2 diabetes

\begin{tabular}{|c|c|c|c|c|c|c|c|c|c|c|}
\hline SNP & Chr & $\begin{array}{l}\text { Nearest } \\
\text { genes }\end{array}$ & & $\begin{array}{l}\text { Type } 2 \text { diabetes } \\
(n=8,552)\end{array}$ & $\begin{array}{l}\text { Control } \\
(n=2,978)\end{array}$ & Adjustment & $\begin{array}{l}\text { OR }(95 \% \text { Cl) } \\
\text { per allele }\end{array}$ & $P q$ & $\begin{array}{l}\text { Powe } \\
*\end{array}$ & $\dagger^{\dagger}$ \\
\hline \multirow[t]{3}{*}{ rs391300 G/At } & 17 & $S R R$ & $\begin{array}{l}\text { GG/GA/AA } \\
\text { (ratio) }\end{array}$ & $\begin{array}{l}4,964 / 2,830 / 471 \\
(0.6 / 0.34 / 0.06)\end{array}$ & $\begin{array}{l}1,745 / 1,012 / 141 \\
(0.6 / 0.35 / 0.05)\end{array}$ & - & $0.97(0.91-1.04)$ & 0.44 & 0.77 & 1 \\
\hline & & & RAF & 0.772 & 0.777 & $\S$ & $0.94(0.87-1.02)$ & 0.15 & & \\
\hline & & & HWE test & 0.011 & 0.712 & & & & & \\
\hline \multirow[t]{2}{*}{ rs17584499 C/T† } & 9 & PTPRD & $\begin{array}{l}\text { TT/TC/CC } \\
\text { (ratio) }\end{array}$ & $\begin{array}{l}187 / 2,105 / 6,051 \\
(0.02 / 0.25 / 0.73)\end{array}$ & $\begin{array}{l}58 / 719 / 2,135 \\
(0.02 / 0.25 / 0.73)\end{array}$ & - & $1.04(0.96-1.14)$ & 0.34 & 0.72 & 1 \\
\hline & & & HWE test $\neq$ & 0.805 & 0.779 & & & & & \\
\hline
\end{tabular}

Chr, chromosome region; $\mathrm{Cl}$, confidence interval; OR, odds ratio for reported type 2 diabetes risk allele; RAF, Risk allele frequency; SNP, single nucleotide polymorphism. †Bold allele indicates reported risk allele for type 2 diabetes. $¥$ P-values for Hardy-Weinberg equilibrium (HWE) test are presented. §For age, sex and log-transformed body mass index. १Calculated in logistic regression analysis with additive model. ††Power to detect $\alpha=0.05$ assuming the effect is $1.1^{*}$, or given the original report **by Tsai et al., allele frequency for type 2 diabetes in the present study, $10 \%$ population prevalence.

susceptibility to type 2 diabetes in the present Japanese population (rs391300-G: odds ratio $[\mathrm{OR}]=0.97$; 95\% confidence interval [CI] $0.91-1.04 ; \quad P=0.44 ;$ rs17584499-T: OR $=1.04$; 95\% CI 0.96-1.14; $P=0.34$ ). Adjustment for age, sex and logtransformed BMI did not affect the association of these SNPs with the disease (rs391300: $P=0.15, \mathrm{OR}=0.94,95 \%$ CI $0.87-$ 1.02, rs17584499: $P=0.65$, OR $=1.02,95 \%$ CI $0.93-1.13)$. We also analyzed the association using another genetic model; dominant and recessive; however, neither analysis showed a significant association (Table S2). Stratified analysis was carried out because differences in age, sex or BMI between cases and controls might produce type 2 errors even after adjusting for these parameters in the logistic regression analyses. The association of these two SNPs with type 2 diabetes was also not observed in stratified analyses by sex or BMI $(<25$ or $\geq 25)$, age of controls ( $\geq 50$ or $\geq 60$ years) or age of diagnosis $(<40$ or $<50$ years old; Table 3 ). Next, we combined two studies (three stages); our present study data and original data by Tsai et al. ${ }^{14}$ using a random effect model because of a remarkable heterogeneity in effect size of both variants across the studies
(Table 4). The result showed that combining the present data with the original Chinese data did not strengthen the original association, and the associations of these two SNPs were no longer significant. Finally, we obtained more comprehensive association data of these two SNP loci from our previous GWAS data ${ }^{12}$. In this GWAS data, rs391300 showed a nominal association with type 2 diabetes $(P=0.04)$, but the direction of risk allele was opposite to the original Chinese study. We further searched association data of directly genotyped or imputed SNPs around the two SNP loci (linkage disequilibrium [LD] block region including each SNP). Based on the Han Chinese and Japanese LD structures from Hapmap JPT and CHB (http://hapmap.ncbi.nlm.nih.gov/; Rel27 Phase II + III, on NCBI B36 assembly, dbSNP b126), we analyzed directly genotyped or imputed genotype data of 101 and 42 SNPs within rs391300 locus (147 kb) and rs17584499 locus (22 kb), respectively, but we did not find any stronger signal for type 2 diabetes than rs391300. Rs17584499 locus including rs17584499 did not show any association with type 2 diabetes in the GWAS data (Tables S3 and S4).

Table 3 | Stratified analysis for the associations of rs391300 and rs17584499 with type 2 diabetes

\begin{tabular}{|c|c|c|c|c|c|}
\hline Stratification & Sample size (case, control) & \multicolumn{2}{|l|}{ rs391300-G } & \multicolumn{2}{|l|}{ rs17584499-T } \\
\hline Male & $(5,007,1,638)$ & $0.98(0.88-1.08)$ & 0.65 & $1.04(0.92-1.18)$ & 0.55 \\
\hline $\mathrm{BMI}<25$ & $(5,113,2,246)$ & $0.91(0.82-1.004)$ & 0.06 & $1.01(0.90-1.14)$ & 0.87 \\
\hline $\mathrm{BMI} \geq 25$ & $(3,014,679)$ & $0.99(0.85-1.15)$ & 0.92 & $1.05(0.88-1.26)$ & 0.59 \\
\hline Age of control $\geq 50$ years & $(8,127,1,622)$ & $0.97(0.88-1.06)$ & 0.47 & $1.01(0.91-1.13)$ & 0.80 \\
\hline Age of diagnosis $<40$ years & $(912,2,925)$ & $0.90(0.79-1.03)$ & 0.11 & $1.06(0.90-1.23)$ & 0.49 \\
\hline Age of diagnosis $<50$ years & $(2,435,2,925)$ & $0.95(0.87-1.05)$ & 0.33 & $1.07(0.95-1.19)$ & 0.28 \\
\hline
\end{tabular}

All data were adjusted by age, sex and log-transformed body mass index (BMI) including them as co-valuables into the same logistic model. Cases and controls whose clinical data (age, sex and BMI) were not complete were excluded from this analysis $\mathrm{Cl}$, confidence interval; OR, odds ratio. 
Table 4 | Combined meta-analysis for the associations of rs391300 and rs17584499 with type 2 diabetes between the present study and original study ${ }^{14}$

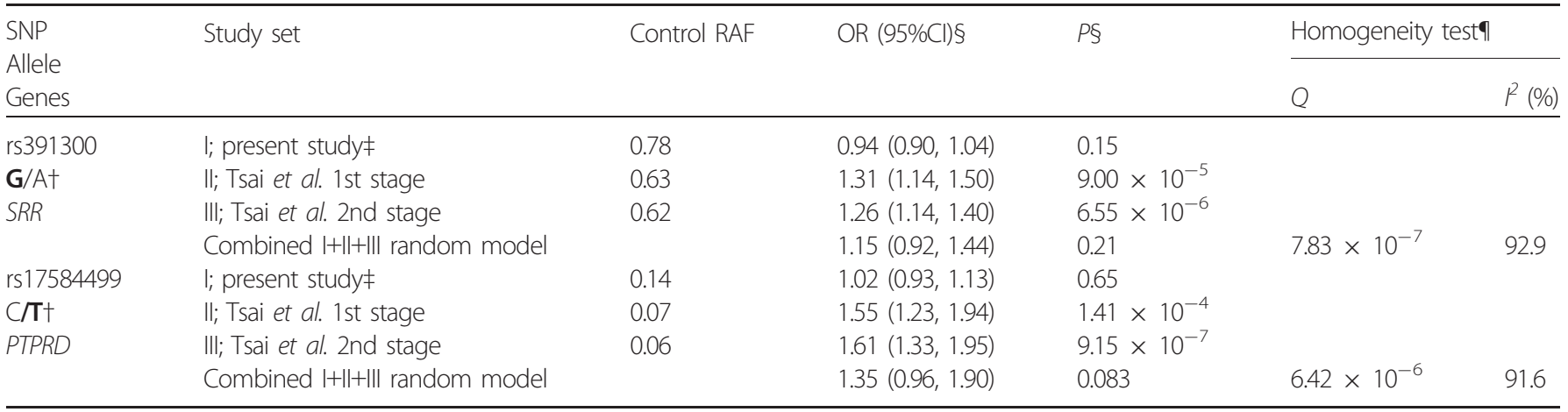

†Bold allele indicates reported risk allele for type 2 diabetes. łAssociation for type 2 diabetes adjusted by age, sex and log-transformed body mass index was used for meta-analysis. §Calculated in logistic regression analysis with additive model. \$Homogeneity of effect size across study groups. $\mathrm{I}^{2}=[\mathrm{Q}$ statistics-(K-1)]/ Q statistics, $\mathrm{K}=$ number of the study. Cl, confidence interval; OR, odds ratio; $\mathrm{Q}$, P-values for Cochran Q statistics; RAF, risk allele frequency; SNP, single nucleotide polymorphism.

Table $\mathbf{5}$ | Association between reported type 2 diabetes risk allele and body mass index†

\begin{tabular}{|c|c|c|c|c|c|c|c|c|}
\hline \multirow[t]{2}{*}{ SNP } & \multicolumn{4}{|c|}{ Type 2 diabetes $(n=8,552)+$ control $(n=2,978)$} & \multicolumn{4}{|c|}{ Control $(n=2,978)$} \\
\hline & $\beta \neq$ & SE & $P$-value & Adjustment & $\beta \neq$ & SE & $P$-value & Adjustment \\
\hline \multirow[t]{2}{*}{ rs391300-G§ } & $-2.56 \times 10^{-4}$ & $2.52 \times 10^{-3}$ & 0.92 & - & $-4.63 \times 10^{-5}$ & $4.44 \times 10^{-3}$ & 0.99 & - \\
\hline & $4.86 \times 10^{-4}$ & $2.48 \times 10^{-3}$ & 0.84 & ब & $1.13 \times 10^{-3}$ & $4.10 \times 10^{-3}$ & 0.78 & †† \\
\hline \multirow[t]{2}{*}{ rs17584499-T§ } & $5.20 \times 10^{-3}$ & $3.00 \times 10^{-3}$ & 0.08 & - & $2.09 \times 10^{-4}$ & $5.27 \times 10^{-3}$ & 0.97 & - \\
\hline & $4.60 \times 10^{-3}$ & $2.94 \times 10^{-3}$ & 0.12 & ब & $2.16 \times 10^{-4}$ & $4.85 \times 10^{-3}$ & 0.96 & †† \\
\hline
\end{tabular}

+Log-transformed values for body mass index were used as the dependent valuables in the linear regression models. $\neq$ Regression coefficient. §Reported risk allele for type 2 diabetes. \Association data were adjusted for age sex and disease state (type 2 diabetes or contol). ††Association data were adjusted for age and sex. SE, standard error; SNP, single nucleotide polymorphism.

Table 6 | Association between reported type 2 diabetes risk allele and glycemic traits

\begin{tabular}{|c|c|c|c|c|c|c|c|c|c|c|}
\hline SNP & \multicolumn{3}{|c|}{$\mathrm{FPG}(\mathrm{mmol} / \mathrm{L})(n=1,332) \dagger$} & \multicolumn{3}{|c|}{ HOMA-IR $(n=900) \dagger$} & \multicolumn{3}{|c|}{ HOMA- $\beta(n=900) \dagger$} & Adjustment \\
\hline \multirow[t]{2}{*}{ rs391300-G§ } & $-8.71 \times 10^{-3}$ & $4.53 \times 10^{-3}$ & 0.055 & 0.03 & 0.031 & 0.33 & 0.052 & 0.031 & 0.10 & - \\
\hline & $-8.15 \times 10^{-3}$ & $4.30 \times 10^{-3}$ & 0.058 & 0.021 & 0.027 & 0.43 & 0.046 & 0.029 & 0.11 & Age, sex, BMl \\
\hline \multirow[t]{2}{*}{ rs17584499-T§ } & $5.86 \times 10^{-3}$ & $5.27 \times 10^{-3}$ & 0.27 & 0.055 & 0.036 & 0.12 & 0.042 & 0.036 & 0.24 & - \\
\hline & $2.20 \times 10^{-3}$ & $5.01 \times 10^{-3}$ & 0.66 & 0.029 & 0.031 & 0.36 & 0.024 & 0.033 & 0.47 & Age, sex, BMl \\
\hline
\end{tabular}

†Log-transformed values for indicated glycemic traits were used as the dependent valuables in the linear regression models. $\neq$ Regression coefficient. §Reported risk allele for type 2 diabetes. BMI, body mass index, FPG, fasting plasma glucose; HOMA- $\beta$, homeostasis model assessment of beta-cell function; HOMA-IR, homeostasis model assessment of insulin resistance; SE, standard error; SNP, single nucleotide polymorphism.

We further examined the association between the two SNPs and quantitative metabolic traits, such as BMI, FPG, HOMAIR and HOMA- $\beta$ (Table 5 and 6; Table S5). We observed a nominal association $(P=0.036)$ between rs391300-G and an increase of HOMA- $\beta$ in the recessive model (Table S5), but any association did not reach statistically significant levels $(P=0.025)$.

\section{DISCUSSION}

In the present study, we carried out a replication study for the association of two variants, rs391300 in SRR and rs17584499 in PTPRD, identified by Chinese GWAS with type 2 diabetes using 11,530 Japanese individuals.

Rs17584499 in PTPRD, the strongest signal in the original Chinese $\mathrm{GWAS}^{14}$, was subsequently shown to be associated 
with progression to type 2 diabetes in a Chinese prospective cohort $^{21}$. PTPRD belongs to the receptor type IIA (R2A) subfamily of protein tyrosine phosphatases (PTPs), which has been implicated in neural development, cancer and diabetes ${ }^{22}$. Rs391300 is located in SRR, which encodes a serine racemase that synthesizes D-serine from L-serine ${ }^{23,24}$; dysregulation of Dserine could affect insulin or glucagon secretion in the pathogenesis of type 2 diabetes ${ }^{25,26}$. The association of rs391300 with type 2 diabetes also showed that rs391300-T had some effects on conferring susceptibility to gestational diabetes or on increasing FPG in Chinese women ${ }^{27}$. Although the effects of these two variants on type 2 diabetes susceptibility have been almost confirmed in Han Chinese populations, a recently carried out meta-analysis using Chinese and other East Asian populations failed to replicate the association of the SNPs with type 2 diabetes $(P>0.01)^{28}$, suggesting the existence of heterogeneity in the effect of these loci between Han Chinese and other East Asian populations, including Japanese.

In the present study, we showed that both rs17584499 and rs391300 were not associated with type 2 diabetes in our Japanese sample. As the present study had sufficient power to detect true association of these SNPs (Table 2), it is not likely that the present results were affected by type 2 error. It seems that there is no obvious difference in clinical characteristics between our present study and the original study (Table S6); therefore, it is unlikely that phenotypic heterogeneity is a principal cause for the discrepancy between the present study and the original Chinese study. As shown in Table 4, risk allele of rs391300 and rs17584499 were quite different between the Japanese and the Han Chinese populations (rs391300-G; 0.78 vs $0.62-0.63$, rs17584499-T; 0.14 vs $0.06-0.07$, Japanese controls in the present study vs Han Chinese controls in the previous report $\left.{ }^{13}\right)$. In addition, there is significant heterogeneity in effect size (odds ratio) between the present study and the study by Tsai et al. (Table 4). Therefore, the lack of an association between the two SNPs and type 2 diabetes in the Japanese population might be explained by the presence of ethnic differences.

Assuming that $\mathrm{LD}$ structures for these two loci in Japanese populations are different from those in Han Chinese populations, the possibility is also present that rs391300 and rs17584499, which reflected the causal signal in the Han Chinese, did not link to the true signals in our Japanese population. To make this point clear, efforts to find true causal variants in these genetic loci, including a fine mapping using the Han Chinese populations, would be required.

From the findings in the present study and the previous studies, it is evident that there are small but significant genetic differences between the Japanese and other East Asian populations. Therefore, we should be more careful when combining Japanese data with those in other East Asian populations. In addition, it should be more emphasized that efforts to increase sample size in a single ethnic group; that is Japanese alone, are important and useful to identify additional novel susceptibility loci in the human genetic study.
In conclusion, we carried out a replication study for the association of rs391300 in SRR and rs17584499 in PTPRD with type 2 diabetes in a Japanese population, and demonstrated that these two SNPs did not show a significant effect on conferring susceptibility to the disease in the Japanese population. The present results indicate that genetic heterogeneity might exist between the Japanese and the Han Chinese populations, although these two East Asian populations are considered to be genetically closer than populations of European or African origin.

\section{ACKNOWLEDGMENTS}

We thank technical staff at the Laboratory for Endocrinology and Metabolism, RIKEN Center for Genomic Medicine, for their technical assistance. This work was partly supported by a grant from the Ministry of Education, Culture, Sports, Science and Technology, Japan. The authors declare that there is no duality of interest associated with this manuscript.

\section{REFERENCES}

1. Billings $L K$, Florez $L C$. The genetics of type 2 diabetes: what we learned from GWAS? Ann N Y Acad Sci 2010; 1212: 59-77.

2. Imamura M, Maeda S. Genetics of type 2 diabetes: the GWAS era and future perspectives. Endocr J 2011; 58: 723-739.

3. Frayling TM. Genome-wide association studies provide new insights into type 2 diabetes aetiology. Nat Rev Genet 2007; 8: 657-662.

4. Unoki $\mathrm{H}$, Takahashi $\mathrm{A}$, Kawaguchi $\mathrm{T}$, et al. SNPs in KCNQ1 are associated with susceptibility to type 2 diabetes in East Asian and European populations. Nat Genet 2008; 40: 1098-1102.

5. Yasuda K, Miyake K, Horikawa Y, et al. Variants in KCNQ1 are associated with susceptibility to type 2 diabetes mellitus. Nat Genet 2008; 40: 1092-1097.

6. Yamauchi T, Hara K, Maeda S, et al. A genome-wide association study in the Japanese population identifies susceptibility loci for type 2 diabetes at UBE2E2 and C2CD4A-C2CD4B. Nat Genet 2010; 42: 864-868.

7. Omori S, Tanaka Y, Takahashi A, et al. Association of CDKAL1, IGF2BP2, CDKN2A/B, HHEX, SLC3OA8, and KCNJ11 with susceptibility to type 2 diabetes in a Japanese population. Diabetes 2008; 57: 791-795.

8. Hayashi T, Iwamoto Y, Kaku K, et al. Replication study for the association of TCF7L2 with susceptibility to type 2 diabetes in a Japanese population. Diabetologia 2007; 50: 980-984.

9. Horikoshi M, Hara K, Ito C, et al. A genetic variation of the transcription factor 7-like 2 gene is associated with the risk of type 2 diabetes in the Japanese population. Diabetologia 2007; 50: 747-751.

10. Onuma H, Tabara Y, Kawamoto R, et al. The GCKR rs780094 polymorphism is associated with susceptibility of type 2 diabetes, reduced fasting plasma glucose levels, increased 
triglycerides levels and lower HOMA-IR in Japanese population. J Hum Genet 2010; 55: 600-604.

11. Ohshige T, Iwata M, Omori $S$, et al. Association of new loci identified in European genome-wide association studies with susceptibility to type 2 diabetes in the Japanese. PLoS ONE 2011; 6: e26911.

12. Imamura M, Maeda S, Yamauchi T, et al. A singlenucleotide polymorphism in ANK1 is associated with susceptibility to type 2 diabetes in Japanese populations. Hum Mol Genet 2012; 21: 3042-3049.

13. Shu XO, Long J, Cai Q, et al. Identification of new genetic risk variants for type 2 diabetes. PLoS Genet 2010; 6: E1001127.

14. Tsai FJ, Yang CF, Chen CC, et al. A genome-wide association study identifies susceptibility variants for type 2 diabetes in Han Chinese. PLoS Genet 2010; 6: e1000847.

15. Imamura $M$, Iwata $M$, Maegawa $H$, et al. Genetic variants at CDC123/CAMK1D and SPRY2 are associated with susceptibility to type 2 diabetes in the Japanese population. Diabetologia 2011; 54: 3071-3077.

16. Kashiwagi A, Kasuga M, Araki E, et al. International clinical harmonization of glycated hemoglobin in Japan: From Japan Diabetes Society to National Glycohemoglobin Standardization Program values. J Diabetes Invest 2012; 3: $39-40$.

17. Alberti KG, Zimmet PZ. Definition, diagnosis and classification of diabetes mellitus and its complications Part 1: diagnosis and classification of diabetes mellitus provisional report of a WHO consultation. Diabet Med 1998; 15: 539-553.

18. The Examination Committee of Criteria for Obesity Disease In Japan. New Criteria for 'Obesity disease in Japan'. Circ J 2002; 66: 987-992.
19. Maeda S, Tsukada S, Kanazawa A, et al. Genetic variations in the gene encoding TFAP2B are associated with type 2 diabetes mellitus. J Hum Genet 2005; 50: 283-292.

20. Nielsen DM, Ehm MG, Weir BS. Detecting marker-disease association by testing for Hardy-Weinberg disequilibrium at a marker locus. Am J Hum Genet 1998; 63: 1531-1540.

21. Chang YC, Chiu YF, Liu PH, et al. Replication of genomewide association signals of type 2 diabetes in Han-Chinese in a prospective cohort. Clin Endocrinol (Oxf). 2012; 76: 365-372.

22. Chagnon MJ, Uetani N, Tremblay ML. Functional significance of the LAR receptor protein tyrosine phosphatase family in development and diseases. Biochem Cell Biol 2004; 82: 664-675.

23. Wolosker H, Blackshaw S, Snyder SH. Serine racemase: a glial enzyme synthesizing D-serine to regulate glutamate$\mathrm{N}$-methyl-D-aspartate neurotransmission. Proc Natl Acad Sci USA 1999; 96: 13409-13414.

24. Wolosker H, Sheth KN, Takahashi M, et al. Purification of serine racemase: biosynthesis of the neuromodulator D-serine. Proc Natl Acad Sci USA 1999; 96: 721-725.

25. Gonoi T, Mizuno N, Inagaki N, et al. Functional neuronal ionotropic glutamate receptors are expressed in the non-neuronal cell line MIN6. J Biol Chem 1994; 269: 16989-16992.

26. Inagaki N, Kuromi H, Gonoi T, et al. Expression and role of ionotropic glutamate receptors in pancreatic islet cells. Faseb J 1995; 9: 686-691.

27. Wang $Y$, Nie M, Li W, et al. Association of six single nucleotide polymorphisms with gestational diabetes mellitus in a Chinese population. PLOS ONE 2011; 6: e26953.

28. Cho YS, Chen C-H, Hu C, et al. Meta-analysis of genomewide association studies identifies 8 new loci for type 2 diabetes in East Asians. Nat Genet 2012; 44: 67-72.

\section{SUPPORTING INFORMATION}

Additional Supporting Information may be found in the online version of this article:

Table S1 | Sequence for primers and probes for multiplex polymerase chain reaction invader assay.

Table S2 | Associations of rs391300 and rs17584499 with type 2 diabetes under a dominant or recessive model.

Table S3 | Association of single nucleotide polymorphisms in rs391300 locus with type 2 diabetes.

Table S4 | Association of single nucleotide polymorphisms in rs17584499 locus with type 2 diabetes.

Table S5 | Association between reported type 2 diabetes risk allele and glycemic traits under a dominant or recessive model.

Table S6 | Clinical characteristics of the present study and original study by Tsai et al. 